\title{
Relación entre el sistema de acomodación, el sistema de vergencias y los problemas de lecto-escritura en los niños de segundo a cuarto de primaria de un colegio de Bogotá
}

\author{
Sandra Bibiana Páez ${ }^{1}$ y Yadira Helena Perea² \\ 1. Optómetra Universidad de la Salle, Msc en Administración Pontificia Universidad Javeriana. \\ Docente Investigador Universidad Antonio Nariño. \\ 2. Optómetra Universidad Antonio Nariño \\ Correspondencia: revistanova@unicolmayor.edu.do \\ Recibido: 24-04-2007 / Aceptado: 16-05-2007
}

\begin{abstract}
Resumen
La amplitud de acomodación y la vergencia son dos factores de la habilidad funcional visual, que al estar alterados influyen frecuentemente en los escolares hasta el punto de ser condiciones en el aprendizaje de la lectoescritura. El objetivo principal de esta investigación fue demostrar que estas alteraciones influyen en el desempeño lecto-escritor. Este estudio descriptivo y correlacional, tomó como población escolar niños entre 7 y 9 años de edad de segundo a cuarto grados de primaria de un colegio de Bogotá. Respetando los criterios de inclusión, se les evaluaron las habilidades visuales de acomodación y de vergencia así como la lectura y la escritura. Mediante la prueba chi cuadrado se logró demostrar que existe relación entre las variables. Este trabajo corresponde a la línea de investigación de Problemas de Aprendizaje desarrollada por la Facultad de Optometría de la Universidad Antonio Nariño. Sus resultados permitieron, entre otras cosas, hacer ajustes a protocolos e instrumentos de variables, con el fin de tenerlos en cuenta en proyectos futuros.
\end{abstract}

Palabras Claves: acomodación, dioptrías, diplopía, insuficiencia de convergencia, reservas fusionales, vergencia.

\begin{abstract}
The amplitude of accommodation and the vergence are two factors of the visual functional ability that, when altered, influence the students frequently to the point of being conditions in the learning of reading and writing. The primary target of this investigation was to demonstrate that these alterations influence in the performance in reading and writing. This descriptive and corelational study took as scholastic population children between 7 and 9 years from second to fourth grade from a Bogota school. They were evaluated in reading and writing, respecting the inclusion criteria, the visual abilities of accommodation, and vergence. By means of the test chi square, it was possible to demonstrate that there is a relation between the variables. This work corresponds to the line of investigation of problems of learning developed by the Faculty of Optometry of the University Antonio Nariño. Their results allowed, among other things, to make adjustments to protocols and instruments of variables to keep them into account in future projects.
\end{abstract}

Key words: accommodation, diopter, diplopia, insufficiency of convergence, fusion reserves, vergence. 


\section{Introducción}

Los ojos son la ventana al mundo, lo cual implica que las alteraciones en el sistema visual necesariamente entorpecen la percepción de la información. A nivel escolar gran porcentaje de la información se recibe mediante estímulos visuales principalmente en las tareas de lectura y escritura. Estudios anteriores han demostrado que el fracaso escolar, no sólo depende de una agudeza visual deficiente o de un error refractivo, se requiere considerar otras habilidades visuales, como son la amplitud de acomodación y vergencias, que al estar alteradas suelen ocasionar sintomatología como cefalea, fotofobia, lagrimea, ardor y cansancio al leer o escribir (1-8).

\section{Acomodación}

La acomodación es un mecanismo flexible, el ojo puede modificar su poder de refracción, o dicho de otra manera, es la capacidad que tiene el ojo de enfocar objetos ubicados a diferentes distancias (9). La acomodación se estudia teniendo en cuenta su amplitud por medio de los métodos convencionales de Sheard, Donders y Jackson. Se ha sugerido que una amplitud de acomodación disminuida puede ocasionar problemas de lectoescritura. El motivo principal es porque incluye sintomatología como visión borrosa, astenopía, cansancio, y somnolencia $(6,10-12)$.

Claudia Jiménez y Luis Alberto García, optómetras de la Universidad de La Salle, probaron en su trabajo de grado, que tanto clínica como estadísticamente, la acomodación disminuida, incide en el rendimiento académico, ya que la lectoescritura mejoró después de realizar el tratamiento ortóptico a 27 escolares de 7 a 11 años de edad (5). Adicionalmente, puede suceder que la amplitud se encuentre diferente en ambos ojos, tal como lo demostró René Cruz, optómetra de la Universidad de La Salle, el cual encontró problemas de Anisoacomodación (3).

Existen otros desórdenes en la acomodación que pueden afectar el proceso de aprendizaje de los escolares (13-16), como son:
* Exceso de acomodación: cuando la amplitud presenta valores mayores con respecto a la edad del sujeto. Los pacientes más afectados son los escolares hipermétropes que aún conservan buena agudeza visual tanto en visión lejana como en la próxima.

* Espasmos de acomodación: anomalía caracterizada por un aumento de la acomodación, alcanzando niveles muy elevados, se atribuye a la sobreacción del músculo ciliar causada por demasiado trabajo en la visión próxima.

* Insuficiencia de acomodación: anomalía en la cual la amplitud de acomodación está por debajo de los valores normales para la edad del individuo.

* Inercia de acomodación: anomalía en la cual hay un retardo para enfocar de visión próxima a visión lejana y viceversa. Es más frecuente en pacientes mayores présbitas y en niños ambliopes con fijación excéntrica.

\section{Movimientos oculares de vergencias}

Las vergencias son movimientos disyuntivos de los ojos que mantienen la visión binocular a cualquier distancia y cuya finalidad es conferir a las personas la habilidad para fijarse en puntos a distintas distancias en el campo visual (11). Estos movimientos se caracterizan porque modifican la posición relativa de los ejes visuales, es decir, los ojos se mueven en dirección contraria. En estos movimientos binoculares hay pérdida del paralelismo de los ejes oculares, existe un cruzamiento de los ejes por delante (convergencia) o por detrás (divergencia) de los ojos (17).

El movimiento de vergencia más empleado es la convergencia, que está asociada a la acomodación, y en este mecanismo la principal falla es la Insuficiencia de convergencia, en la que existe incapacidad para obtener o mantener la coincidencia de los ejes visuales sobre un objeto de fijación próximo. Se manifiesta por un punto próximo de convergencia alejado y una amplitud de vergencias bajas. El paciente con insuficiencia de 
convergencia presentará astenopia, pérdida del renglón, cefaleas, ardor, lagrimeo, somnolencia, salto de letras y ocasionalmente diplopía, estas condiciones influyen en el rendimiento escolar, como fue anotado por Claudia Jiménez y Luís Alberto García, optómetras de la Universidad de La Salle (5).

Zoilo Cuellar, en 1974, señaló que las alteraciones de la binocularidad coinciden con las dificultades en la lectura y van a producir una descoordinación de la secuencia pausa-sacudida (movimientos sacádicos) durante la lectura. Ésta pérdida de la coordinación dificultará, notablemente el aprendizaje de los símbolos escritos, al niño que comienza sus estudios, constituyendo un serio obstáculo en la vía visual de la percepción. Con respecto a este problema, Lucia Sanín de Gutiérrez, en 1977, anota que los problemas de binocularidad alteran la coordinación ojo mano, que pueden ser la causa de problemas del aprendizaje tales como el cambio de lugar de un símbolo, salto de renglones, entre otras (18).

Además, se ha mencionado que hasta dos tercios de los que padecen trastornos específicos de aprendizaje suelen manifestar dificultades en localizar letras con precisión, como lo concluyó Fowler, aplicando el test Dunlop, que consiste en un estudio de las reservas fusionales positivas y negativas y la apreciación subjetiva de la inestabilidad de las imágenes antes del momento de la ruptura de fusión. La subjetividad de la prueba dificulta las conclusiones.

\section{Competencias lecto-escritoras}

Los principales signos encontrados tanto en la lectura como en la escritura, y que fueron evaluados en el presente estudio, se resumen de la siguiente manera: en la lectura se puede presentar los siguientes signos: salto del renglón, regresiones, usar el dedo para guiarse, movimientos asociados de cabeza, sensación de movimientos de líneas, poca capacidad para recordar lo que se lee, velocidad lectora lenta, poca concentración, no se utilizan los signos de puntuación y malas posturas al leer $(3,18-21)$.

\section{En escritura se presenta:}

* Rotación: confusión de letras con formas similares, tanto al leerlas como al escribirlas (por ejemplo, "b” - “d” - “p” -“q”).

* Inversiones: modificación de la secuencia correcta de las sílabas reflejada al leer y/o escribir (por ejemplo, “la" - "al”). Es normal que se produzcan este tipo de errores hasta el primer curso de primaria, deben ser poco frecuentes en el segundo curso y cesar en el tercero.

* Confusiones: cambiar una letra por otra, ya sea gráfica o fonéticamente, por ejemplo, “b” - “p” - “t” - “d”.

* Omisiones en lectura y escritura: supresión de una o varias letras en la palabra (por ejemplo, "soldado" - “sodado”), ausencia de fonemas ("va” en lugar de “van”), de sílabas (“panaría” - "panadería”) y en línea (salto del renglón).

* Agregados o adiciones: añadir fonemas, letras, sílabas o palabras inexistentes, leyendo y/o escribiendo (por ejemplo, “estos” en lugar de “esto”).

* Contaminaciones: cuando una sílaba o palabra escrita o leída fuerza a otra y se confunde con ésta, resultando una mezcla de ambas ("pájaro rojo” = "pajajo”).

* Sustituciones: se cambian fonemas ("las" por "los"), sílabas (“es” por “de”) y palabras (“acabado” por “atado”).

* Disociaciones: fragmentar una palabra de forma incorrecta cuando se lee y/o escribe.

* El manejo inadecuado del renglón, la mala separación de las palabras o sílabas (Figura 1).

\section{Materiales y métodos}

\section{Tipo de estudio:}

* Descriptivo simple: para mostrar cual es la distribución de la población teniendo en cuenta las variables estudiadas y compararlas en los dos grupos estudiados.

* Correlacional: para evaluar la hipótesis de trabajo a cerca de la relación entre las variables: acomodación y vergencias y el desempeño en lecto-escritura. 
60

NOVA - PUBLICACIÓN CIENTÍFICA - ISSN:1794-2470 VOL.5 No. 7 ENERO - JUNIO DE 2007:1-100

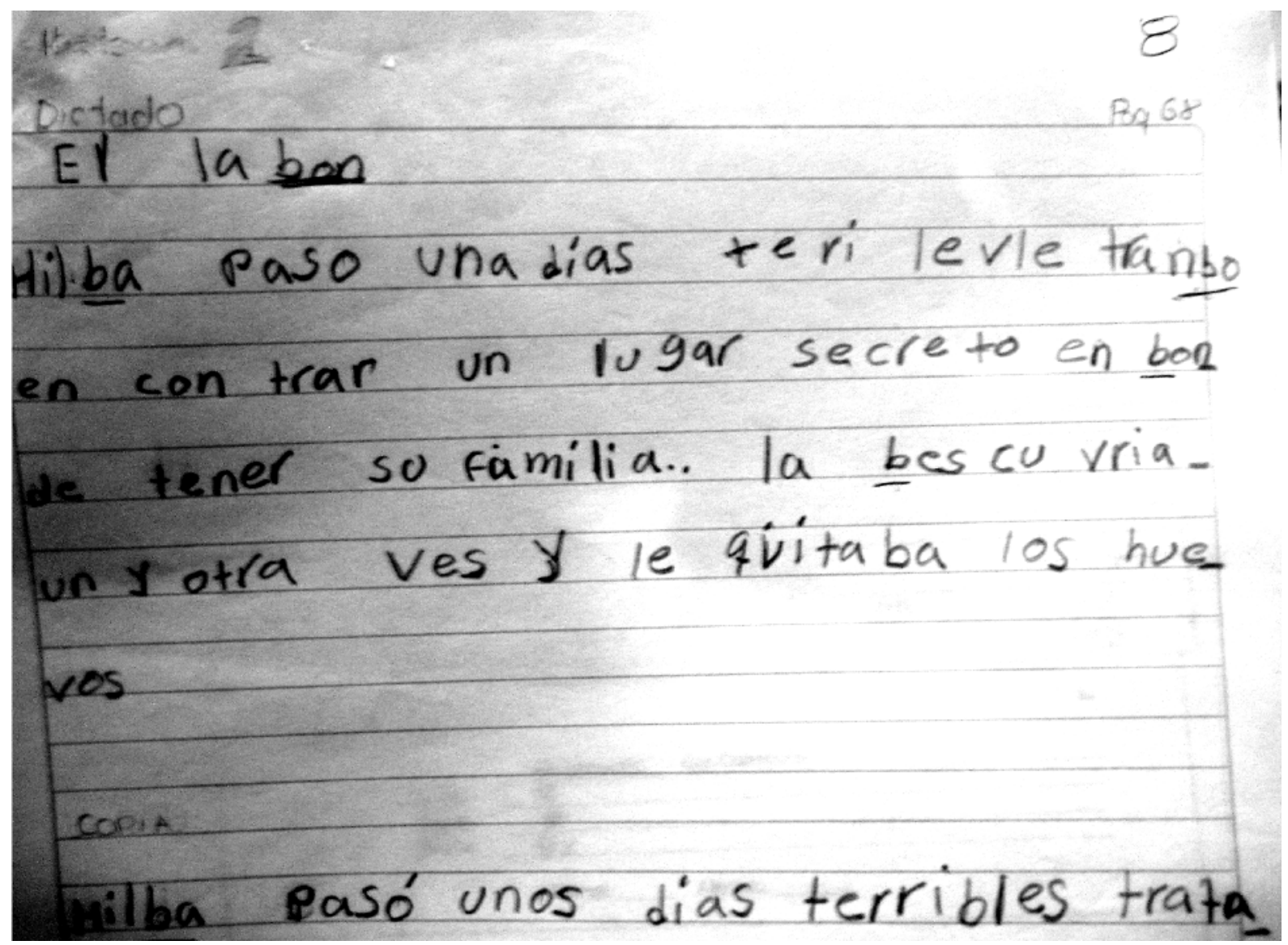

Figura 1. Muestra del manejo inadecuado del renglón.

Población y muestra

De una población de un colegio de Bogotá, se seleccionó una muestra con los siguientes criterios de inclusión: niños entre los 7-9 años con agudezas visuales no inferiores a 20/25 en cada ojo y 0,5 de cerca, emétropes y con hipermetropías no mayores a +0.75 dioptrías y sin tropias. Se realizó la evaluación de la acomodación, las vergencias y la lecto-escritura, y se dividió la muestra en dos grupos: el grupo de estudio, formado por los estudiantes con problemas de lectoescritura y el grupo control, formado por escolares con buen desempeño en este aspecto. Se utilizó la prueba chi-cuadrado para el análisis estadístico.

Los parámetros que se tuvieron en cuenta en estudio fueron:

* Amplitud de Acomodación (A.A): utilizando la técnica de Sheard se denominó como normal los hallazgos mayores o iguales a 11 dioptrías y alterada, si se encuentran menores a este valor (6).

* Prisma Cover test: utilizando la medida realizada en dioptrías prismáticas, con prismas base interna para las forias o tropias tipo exo y base externa para las endo. Cuando la medida del prisma es mayor en visión próxima y esta diferencia es mayor a 10 prismas nos encontramos frente a un diagnóstico de insuficiencia de convergencia.

Reservas fusionales positivas y negativas (RFP y RFN). Se midió activando la RFP por medio de prismas base externa y de la RFN mediante prismas base interna hasta inducir diplopía o visión doble. Se evaluaron tanto en visión lejana (VL) y visón próxima (VP). Los valores de normalidad se encuentran en la Tabla 1 (17). 
Tabla 1. Valores normales de reservas fusionales.

\begin{tabular}{ll}
\hline & VALORES NORMALES RF \\
Visión lejana. & RFP: $20-25$ Dpt $\Delta$ \\
& RFN: $6-8$ Dpt $\Delta$ \\
Visión próxima & RFP: $30-35$ Dpt $\Delta$ \\
& RFN: $10-12$ Dpt $\Delta$ \\
\hline
\end{tabular}

* Punto Próximo de Convergencia (PPC): es el punto más cercano donde los ojos pueden converger sin perder la binocularidad. Tomado con luz se considera normal cuando corresponde a un valor menor o igual a $10 \mathrm{~cm}$. y alterado si es mayor a este valor.

* Lecto-escritura: el diagnóstico de buen o mal desempeño en lecto-escritura fue realizado por la disciplina de Psicología, y se valoró de la siguiente manera: lectura oral, encontrándose que cuando existe mal desempeño existe una velocidad lectora lenta y errores al leer como regresiones, confusiones, pérdida del renglón, inversiones, etc. En la escritura, evaluada en la copia como en el dictado, se observó la presencia de: omisiones, disociaciones, rotaciones, confusiones, contaminaciones, etc., que fueron evaluados como mal desempeño.

\section{Recolección de datos}

Todos los datos obtenidos en este estudio fueron consignados en la historia clínica de cada uno de los participantes.

\section{Resultados}

\section{Distribución de la muestra según los hallazgos encontrados}

De una población de 265 escolares en edades comprendidas entre 7 y 9 años, se escogió una muestra de 36 teniendo en cuenta los criterios de inclusión descritos en la metodología. El mayor porcentaje correspondió al grupo de 9 años (38\%), seguido del grupo de 8 años y por último el de 7 años (29\%). La distribución de la muestra según la escolaridad correspondió al 45\%, 33\%

y 22\% a los grados cuarto, tercero y segundo respectivamente. En la Figura 2 se muestra la distribución según los hallazgos en lecto-escritura.

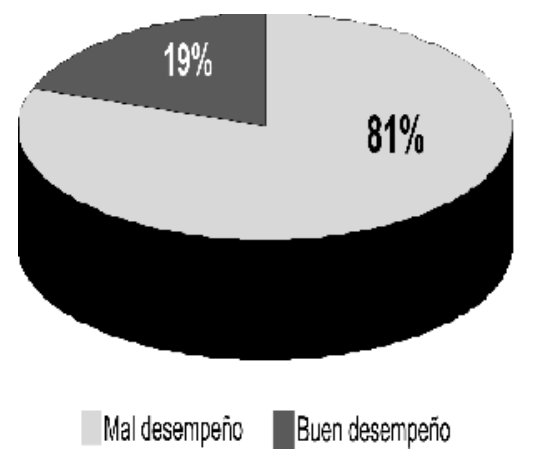

Figura 2. Distribución de la muestra según el desempeño en lectoescritura

Los principales errores cometidos por los escolares con mal desempeño en lectura se muestran en las Tablas 2 y 3, los principales errores en la escritura cuando se les realiza un dictado.

Tabla 2. Distribución de la muestra según los hallazgos encontrados en la evaluación del desempeño en lectura oral teniendo en cuenta los signos presentados.

SIGNOS

Regresiones

Perdida del renglón

Omisiones

No sigue signos de

puntuación

Confusión

Inversión
Presentan el signo

(\%)

33

31

17

3

3

3 
Tabla 3. Distribución de la muestra según los hallazgos encontrados en la evaluación del desempeño en escritura (dictado y copia) teniendo en cuenta los signos presentados.

\begin{tabular}{l|cc}
\hline & & \multicolumn{2}{c}{ Porcentaje (\%) } \\
\hline SIGNOS & DICTADO & COPIA \\
\hline Ausencia de fonemas & 71 & 46 \\
Omisiones & 71 & 7 \\
Rotaciones & 29 & 50 \\
\hline
\end{tabular}

Comparación de los promedios del grupo de estudio y grupo control.

\section{Amplitud de Acomodación}

En la Tabla 4 se registran los promedios en dioptrías, tanto para el ojo derecho como para el ojo izquierdo, que fueron mejores en los pacientes con buen desempeño en lecto-escritura.

Tabla 4. Valores promedio Amplitud de acomodación

\begin{tabular}{lcc}
\multicolumn{1}{c}{ AMPLITUD DE } & $\begin{array}{c}\text { BUEN DESEMPEÑO EN } \\
\text { LECTO-ESCRITURA }\end{array}$ & $\begin{array}{c}\text { MAL DESEMPEÑO EN LECTO- } \\
\text { ESCRITURA }\end{array}$ \\
AMPLITUD DE & & \\
ACOMODACIÓN OJO & 10.96 & 8.24 \\
$\begin{array}{l}\text { DERECHO } \\
\text { (A.A.OD) }\end{array}$ & & \\
AMPLITUD DE \\
ACOMODACION OI \\
(AAOI)
\end{tabular}

\section{Reservas fusionales positivas y negativas}

En la Tabla 5 se observa que los promedios en la medida de RFP en visión próxima, se encuentran disminuidos en los escolares con mal desempeño en lecto-escritura.

\begin{tabular}{ccc}
\hline & Tabla 5. Valores promedios reservas fusionales. \\
\hline RESERVAS FUSIONALES & BUEN DESEMPEÑO EN LECTO- & MAL DESEMPEÑO EN LECTO- \\
& ESCRITURA & ESCRITURA \\
RFP VP & 30,57 & 22.93 \\
\hline
\end{tabular}

\section{Prisma Cover Test en visión próxima}

Los escolares con buen desempeño en lecto-escritura, obtuvieron en promedio para visión próxima, 6 prismas base interna, mientras que el promedio para los escolares con mal desempeño fue de 8.93 prismas base interna. Lo anterior indica que la exoforia es mayor en el grupo de estudio.

\section{Punto próximo de convergencia con luz}

En la Figura 3 se muestra los valores del PPC con luz para ambos grupos.

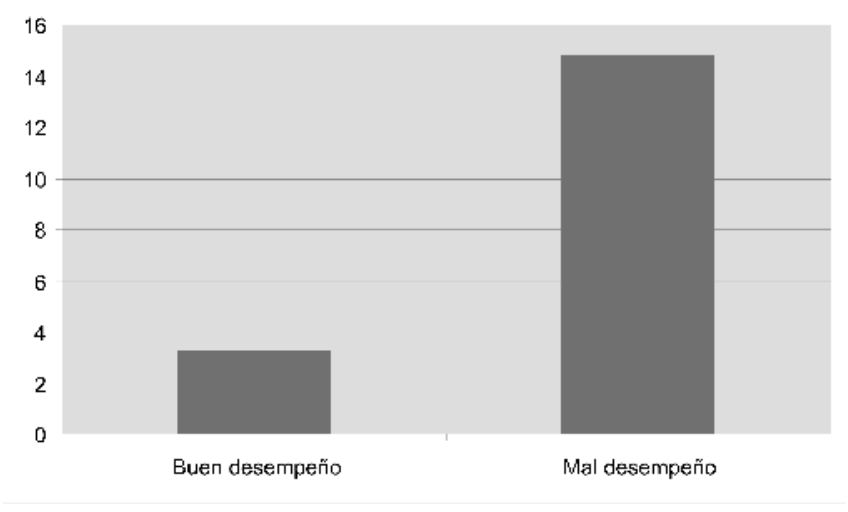

Figura 3. Valores promedio PPC con luz. 
Tabla 6. Valores promedio de velocidad y número de errores en la evaluación del K-D Test

\begin{tabular}{lcc}
\hline & BUEN & \\
MOVIMIENTOS SACADICOS & DESEMPEÑO & MAL DESEMPEÑO \\
VELOCIDAD & $72^{\prime \prime}$ & $1 ' 22^{\prime \prime}$ \\
NÚMERO DE ERRORES & 2 & 11 \\
\hline
\end{tabular}

Tabla 7. Valores promedio de velocidad y número de errores en la evaluación de la lectura oral.

\begin{tabular}{lcc}
\multicolumn{1}{c}{ LECTURA ORAL } & BUEN & MAL \\
\hline VELOCIDAD & DESEMPEÑO & DESEMPEÑO \\
\hline NÚMERO DE ERRORES & $43^{\prime \prime}$ & $1 ' 22^{\prime \prime}$ \\
\hline
\end{tabular}

\section{Movimientos sacádicos.}

En la Tabla 6 se muestra que los escolares con mal desempeño en lecto-escritura se demoraron más, en promedio, en la ejecución de la prueba y tuvieron mayor número de errores.

\section{Lectura}

En la Tabla 7, muestra los valores promedio en velocidad y número de errores cometidos en la lectura oral, estos datos fueron mayores en los estudiantes con mal desempeño en lecto-escritura.

\section{Relación entre las variables}

Al aplicar la prueba chi-cuadrado, y comparar las frecuencias entre el grupo de estudio y control, con un 95\% de confiabilidad y 2 grados de libertad, se encontró que existe relación entre las alteraciones de acomodación, reservas fusionales en visión próxima, punto próximo de convergencia con luz, movimientos sacádicos e insuficiencia de convergencia con el mal desempeño en lectura y escritura.

\section{Discusión}

Teniendo como punto de referencia el marco conceptual que existe sobre el tema y los resultados obtenidos en este trabajo, es importante anotar que la agudeza visual y los errores refractivos, no son las únicas que influyen en el aprendizaje de la lecto-escritura. Se logró demostrar estadísticamente, la relación que existe entre la disminución de la amplitud de acomodación y la insuficiencia de convergencia con el rendimiento de la lectoescritura. Lo anterior sugiere la realización de un tratamiento ortóptico, lo cual permitirá corroborar clínicamente la relación entre las variables analizadas.

Al comparar los promedios de la amplitud de acomodación, las reservas fusionales en visión próxima, el punto próximo de convergencia, el covertest de cerca se encontró que en el grupo de estudio se estos se encuentran alterados con respecto a los patrones de normalidad. Además, al igual que en otros estudios, se corroboró en el diagnóstico de Insuficiencia de convergencia, la asociación con las reservas fusionales bajas y el PPC alejado.

Investigaciones anteriores han mostrado que existe relación entre la insuficiencia de convergencia y la alteración de los movimientos sacádicos, aunque no se analizó estadísticamente esta relación, si se encontró que los movimientos sacádicos se encuentran alterados en los pacientes con dificultades en la lectura, en donde la velocidad de ejecución de la prueba es muy lenta y se presentan muchos errores.

Con respecto a la escritura, se presenta menos errores en la copia que en el dictado, lo que sugiere que el escolar puede tener problemas de integración auditiva. Sólo se encontró un caso de anisoacomodación, lo que contradice otros estudios donde reportan en los pacientes el hallazgo 
de diferencias mayores a 2.00 dioptrías de acomodación de un ojo con respecto al otro.

Para terminar, los resultados de este estudio, permitieron ajustar los instrumentos y protocolos de atención a las variables de acomodación y de vergencias, que van a ser utilizadas en futuros proyectos de investigación de esta línea. Además, de hacer dos recomendaciones: la primera, el manejo de los problemas de aprendizaje debe darse mediante un enfoque multidisciplinario, objetivo de la línea de investigación de la Facultad de Optometría de la Universidad Antonio Nariño, en colaboración de disciplinas como Optometría, Medicina, Fonoaudiología, Terapia Ocupacional, Nutrición y Psicología. La segunda, teniendo en cuenta que algunos autores señalan que cuando existe problemas de pérdida de renglón se encuentra alterada la coordinación ojomano, sería interesante corroborar esta relación para futuros estudios.

\section{Agradecimientos}

Directivas del Colegio Salitre San Carlos, Bogotá, Colombia, por permitir la realización del estudio en el plantel educativo. Al Doctor Mario Esteban Bautista Ochoa y Anna Francisca García por su colaboración en parte de este proceso, investigadores de la línea de problemas de aprendizaje. A la Doctora Liliana Tapias, Psicóloga, por su asesoría en los hallazgos en lectoescritura. NOV

\section{Referencias}

1. American Academy of Optometry -American Optometric Association. Declaración institucional conjunta sobre visión, aprendizaje y dislexia. Archivos optométricos, gaceta óptica. Volumen 2, Número 1.
2. Asociación de padres de niños con Dislexia. Dislexia y dificultades de Aprendizaje. Editorial Ciencias de la Educación Preescolar y especial. 1994.

3. Cruz H. Importancia del estudio y tratamiento de las alteraciones visuales sensoriomotoras en niños con problemas específicos del aprendizaje, el uso de las postimagenes, y el concepto de anomalía de a dirección visual espacial y en su reeducación. Tesis. Pregrado en Optometría. Universidad de La Salle. 1980.

4. Díaz S. Bases optométricas para una lectura eficaz. Tesis. Maestría en en Optometría y Entrenamiento Visual. Centro de Optometría Internacional. España. 2004.

5. Jiménez L. Incidencia de la amplitud de acomodación y vergencias en el rendimiento académico en escolares. Tesis de pregrado en Optometría. Universidad de La Salle. 1993.

6. Leiva F. Visión y aprendizaje. Tesis. Pregrado en Optometría. Universidad de La Salle. 1999.

7. Pérez A. Incidencia del estado visual en problemas del aprendizaje: dislexia y discalculia. Tesis. Pregrado en Optometría. Universidad de La Salle. 1997.

8. Rodríguez M. Corrección de los defectos de refracción y mejoramiento de los aspectos conductual, social y de rendimiento escolar en alumnos matriculados en el sector público. Congreso nacional de optometría: mejor salud visual para más comunidades. Medellín: Fedopto. 1994.

9. Castiella J. La refracción en el niño. Editorial Mc Graw Hillinteramericana. Primera edición. España. 1997.

10. Siderov J, DiGuglielmo L. Binocular accommodative facility in prepresbyopic adults and its relation to symptoms. Optom Vis Sci. 1991;68:49-53.

11. Sheard C: Dynamic ocular test. Columbus: Lawrence, reimpreso en The Sheard vol. 1957. Philadelphia: Chilton Book Co. 1917:90-112.

12. Donders F. On the anomalies of accomodation and refraction of the eye. London: New Sydenham Society. 1864:173-225.

13. Avello N. Análisis comparativo entre la medida de forias con dos pruebas en pacientes con reservas bajas. Tesis de pregrado en Optometría. Universidad de la Salle. Bogotá, 1980

14. Borich M. Clinical Refraction. WB Sauders Company. Pensilvania. 1998.

15. Borras M. Visión binocular, diagnóstico y tratamiento. Ed. Alfaomega. 1996.

16. Herrera G. Guías y protocolos de atención en salud visual y ocular para la Facultad de Optometría de la Universidad Antonio Nariño. Tesis de pregrado en Optometría. Bogotá. 2004.

17. Triana G. Estudio piloto para elaborar el test de CA/AC, para confort visual y establecer valores normales de la relación ACA en la población colombiana. Tesis de pregrado en Optometría Universidad de La Salle. 1993

18. Carvajal P. Deficiencias visuales comparadas al bajo rendimiento escolar en niños gamines. Tesis de pregrado en Optometría. Universidad De La Salle. Facultad de Optometría. 1980.

19. Alvarez L, Gonzáles P. Dificultades en la adquisición del proceso lector. Psicothema. 1996;8:573-586.

20. Arango C. Problemas específicos en el aprendizaje: una visión actual. Rev. Universidad de San Buenaventura. 1996;6:43-46.

21. Prieto D. y et. Estrabismo. 3. a edición. La Plata, Bs. 1996.
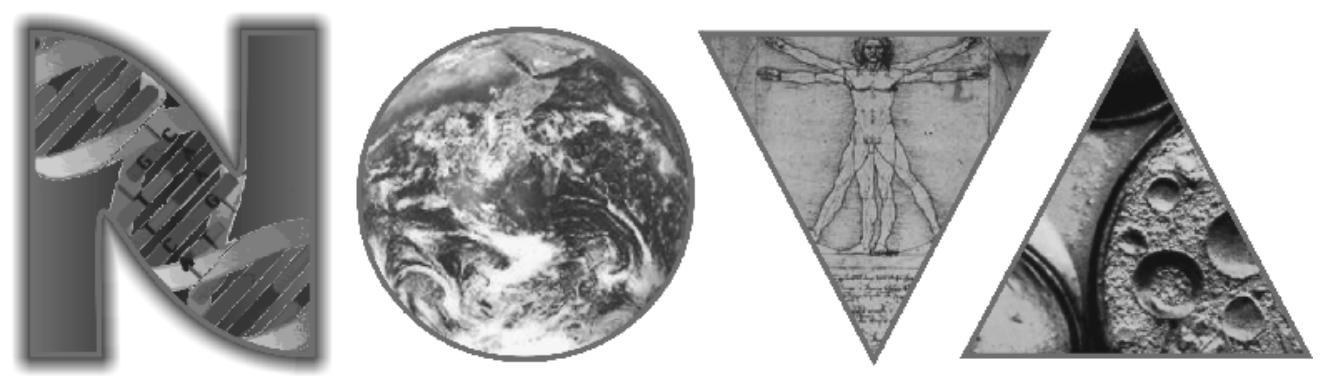